\title{
Chronic Meningitis by Histoplasmosis: Report of a Child with Acute Myeloid Leukemia
}

\author{
Pereira G.H. ${ }^{1}$, Pádua S.S. ${ }^{2}$, Park M.V.F. ${ }^{1}$, Muller R.P. ${ }^{1}$, Passos R.M.A. ${ }^{2}$ and Menezes Y. ${ }^{3}$ \\ ${ }^{1}$ Department of Infectious Disease of Brigadeiro Hospital; ${ }^{2}$ Department of Hematology and Pediatric of Brigadeiro Hospital; ${ }^{3}$ Department of \\ Pathology of Adolfo Lutz Institute; São Paulo, SP, Brazil
}

\begin{abstract}
Meningitis is a common evolution in progressive disseminated histoplasmosis in children, and is asymptomatic in many cases. In leukemia, the impaired of the $\mathbf{T}$ cells function can predispose to the disseminated form. The attributed mortality rate in this case is $20 \%-40 \%$ and the relapse rate is as high as $50 \%$; therefore, prolonged treatment may be emphasized. We have described a child with acute myeloid leukemia (AML), that developed skin lesions and asymptomatic chronic meningitis, with a good evolution after prolonged treatment with amphotericin B deoxycholate followed by fluconazole.

Key-Words: Histoplamosis, chronic meningitis, antifungal therapy.
\end{abstract}

Acute Myeloid Leukemia correlates to $20 \%$ of acute childhood leukemia and M3 subtype correspond to $10 \%$ of all AML.

Infections still remain a major cause of therapy-associated morbidity and mortality in children with AML [1]. Fungi and Gram-positive cocci are the most common organisms associated with infection-related mortality [2].

Given the enormous increase in our understanding of the underlying biology of AML, and the development of many new targeted drugs, it should be possible to achieve highquality cure in nearly all children and adolescents with AML within the next few decades [3,4].

Histoplasma capsulatum infection is endemic in South America, probably due to the conditions of moderate climate, humidity and soil characteristics.

The disseminated histoplasmosis forms occur easily in cases where there are disorders that impair the function of $\mathrm{T}$ cells, monocytes, and macrophages. Additional risk factors include large inoculum exposure and acquired immunodeficiency resulting from use of immunosuppressive agents, malnutrition or HIV infection. Most are ultimately found to be immunologically healthy [5-9]. Central Nervous System (CNS) involvement is recognized in 5\%-10\% of cases of progressive disseminated histoplamosis. [10], and is common in disseminated histoplasmosis of childhood [11]. Clinical syndromes include subacute or chronic meningitis, focal brain or spinal cord lesions, stroke syndromes and encephalitis $[10,12]$. The diagnosis of CNS histoplasmosis can be obtained sometimes by a false-positive result from a non-culture-based test, including the Histoplasma antigen assay. It is important to use multiple tests to reach the diagnosis because there is not a test that exhibits a high sensitivity alone. Histoplasma may be identify in multiple organs and the difficulty stays in cases with CNS involvement [12].

Received on 27 June 2008; revised 20 November 2008.

Address for correspondence: Dr.Graziella Hanna Pereira. Avenida Jandira, 79, apt. 231-Bloco A2. São Paulo, SO, Brazil. E-mail: ghpereira1@terra.com.br. Phone: 5511 9915-8008.

The Brazilian Journal of Infectious Diseases 2008;12(6):555-557. (C) 2008 by The Brazilian Journal of Infectious Diseases and Contexto Publishing. All rights reserved.
The optimal treatment for CNS histoplamosis, the period of treatment and the recommendation of suppressive therapy is unknown $[6,12]$.

We have described a child with AML, that developed skin lesions and chronic meningitis, with good evolution after prolonged antifungal treatment.

\section{Case Report}

We report a 13 year old white man child with diagnosis of AML (M3 subtype) with disseminated nodules at epidermis that were formed and drained for times spontaneously whitish stuff and evolved as crusts (Figure 1). The initial histological diagnosis was leishmaniosis. In the revision of the histological diagnosis were found wounds granulomatous with immunohistochemical stain positive for Histoplasma (Figure 2). It was treated initially with glucantime with partial clinic improvement, and after histological revision with the histoplasmosis diagnosis, the treatment was changed to itraconazole. As part of the clinical investigation for leukemia a cerebral spinal fluid (CSF) was solicited, and revealed a moderate increase in the total cell count (454 cells) with a majority of monocytes and lymphocytes and with a normal protein and glucose range. The magnetic brain resonance was normal and the histoplasmosis serology in serum and CSF was negative. The cultures for Histoplasma from blood, skin and CSF were negatives. The serology for paracoccidioidomycosis, histoplasmosis and aspergillosis were negatives. There were discharged any other causes of SNC diseases, such as: cytomegalovirosis, tuberculosis and herpes. The patient had not been received intrathecal chemotherapy. He was hospitalized, despite of not being symptomatic, for amphotericin B administration during 21 days, occasion where CSF became normal, and the skin lesions became worse. Once again he was submitted to skin lesions biopsy with the result of granulamatous reaction as before, but this time, out of fungus. The patient was followed ambulatory during 1 year, while receiving fluconazole. The initial dose was $400 \mathrm{mg}$ per day, and in the end it was 100 mg per day. The dose agreement was made according to hepatic function. After this year, he did not presented any new skin lesions. 
Figure 1. Patient skin lesions in arms and legs.
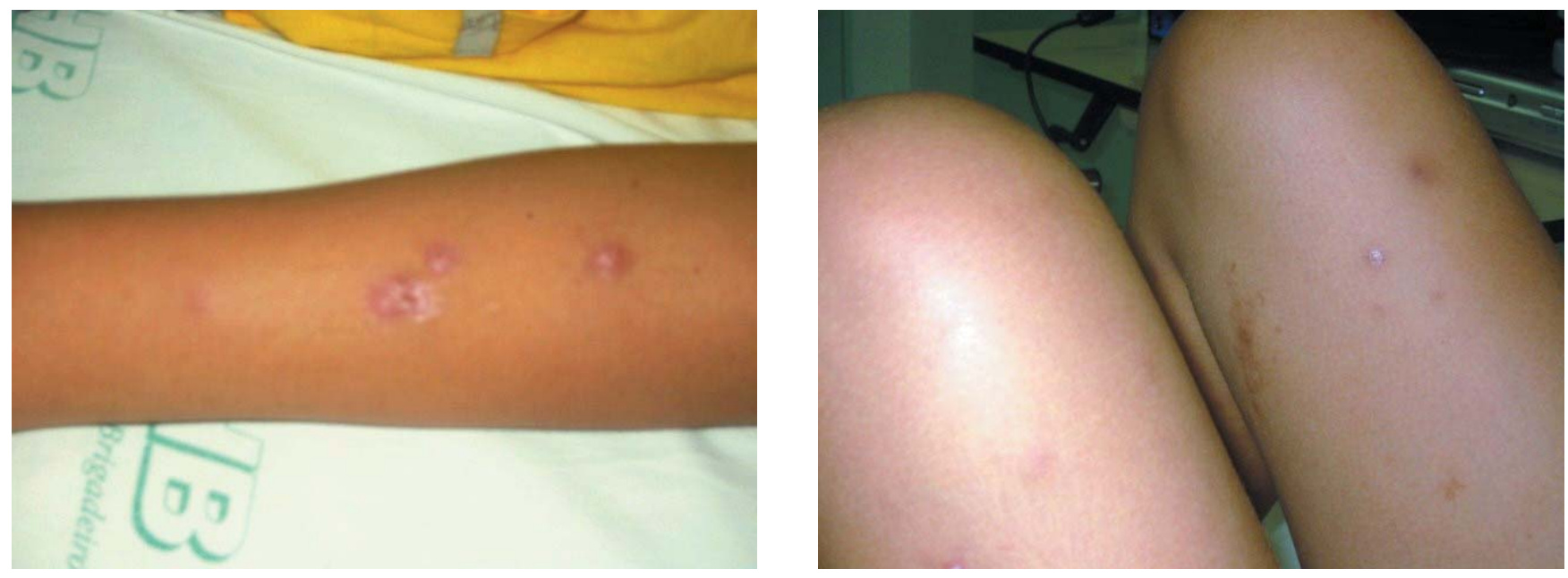

Figure 2. Description of skin pathologic findings. 2.1: Chronic inflammatory granulomatous in skin biopsy. 2.2: Basophilic rounded structures in histiocytes stained by hematoxylin and eosin. 2.3A: Structures stained by Grocott Gomori’s methenamine silver nitrate. 2.3B: Immunohistochemical stain positive for Histoplasma sp. antigen.
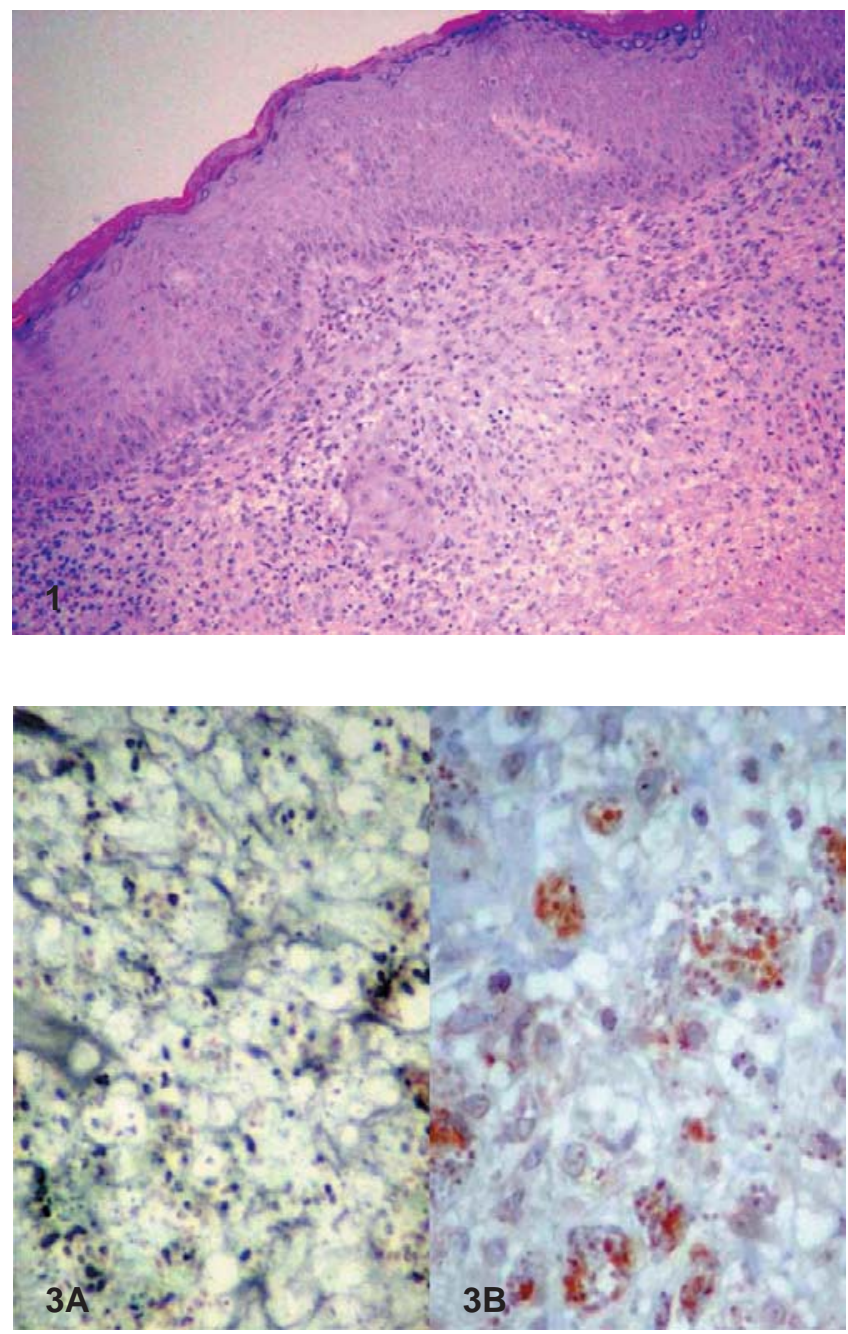

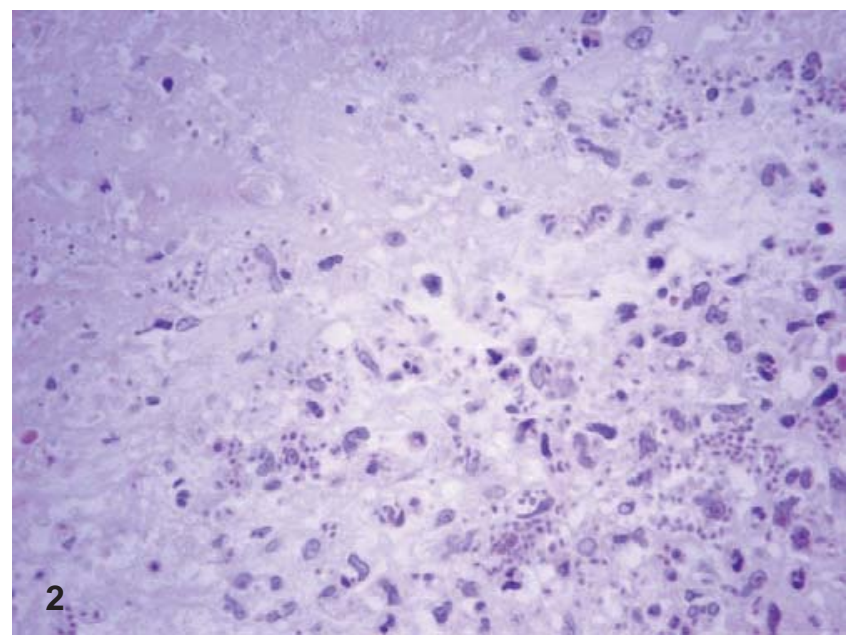

\section{Discussion}

The rare presentation of chronic meningitis by histoplasmosis in children is described in literature. The clinical presentation of CNS histoplasmosis includes meningitis, brain parenchymal lesions and/or spinal cord, or both [10].

Disseminated histoplasmosis should be considered in infants from endemic areas who have fever, hepatosplenomegaly and hematologic abnormalities. These patients develop transient hyperglobulinemia and $\mathrm{T}$ cell deficiency that improve with specific treatment [11].

The high prevalence of cutaneous involvement in Brazilian patients (44.3\%) compared with North American reports (p $<0.01$ ) suggests that different strains of Histoplasma capsulatum may induce different clinical manifestations of the same disease [9]. 
The disseminated form with skin and meningeal involvement can appear in immunosupressed patients and mainly in infants [6]. Our patient was neurological asymptomatic and the meningitis was like an AML typical meningitis.

Histoplasmosis as cause of chronic meningitis in patients with no other evidence of dissemination, is sub-estimated, maybe. Histoplasmosis may also cause cerebral or spinal cord mass lesions resembling cancer or abscesses, and encephalitis. Diagnosis of chronic meningitis or mass lesions caused by Histoplama capsulatum may be difficult and involves careful analysis of serologic tests, cultures and tests for HPA in body fluids [10].

Difficult of specific diagnosis may occur in function of antifungal administration (as itraconazole) and the false positive fungal serology because of immunosupression and/ or quimiotherapy.

In this present case, after administration of the specific treatment, there was a normalization in CSF parameters, but the skin lesions persisted in despite of the absence of fungi at the wounds biopsy. The response to the specific treatment, emphasized the diagnosis.

The response to therapy is inferior than that expected comparing to other types of histoplasmosis; thus, an aggressive approach is recommended. Progressive disseminated histoplasmosis in children is fatal if untreated. Amphotericin B deoxycholate that was given for 4 weeks has been used successfully and with minimal toxicity. A lipid formulation of amphotericin B may be substituted if thepatient is intolerant to amphotericin B deoxycholate. A shorter course of amphotericin B followed by an azole was effective in $74 \%$ of cases and remains an alternative to a prolonged course of amphotericin B treatment. Different from the same cases seen in the population of adults, meningitis that accompanies progressive disseminated histoplasmosis of infancy responds to amphotericin B deoxycholate (given for 4-6 weeks) without ahigh rate of relapse. Treatment recommended forprogressive disseminated histoplasmosis in children does not need to be modified in patients who have CNS involvement [6,12].
Chang et al. in an study of 30 cases of histoplasmosis observed in Mato Grosso do Sul State, Brazil, described an attributed mortality of $40 \%$ [5].

Therefore, we describe a patient with LMA-M3 with a rare presentation of disseminated histoplasmosis, with skin lesions and asymptomatic and chronic meningitis that presented a good clinical evolution after antifungal treatment. In immunosupressed patients we suggest an inquiry of SNC concerning histoplasmosis diagnosis.

\section{References}

1. Lehrnbecher T., et al. Infectious complications in pediatric acute myeloid leukemia: analysis of the prospective multi-nstitutional clinical trial AML-BFM 93. Leukemia 2004;18(1):72-7.

2. Sung L., et al., Microbiologically documented infections and infection-related mortality in children with acute myeloid leukemia. Blood 2007;110(10):3532-9.

3. Kaspers G.J., C.M. Zwaan. Pediatric acute myeloid leukemia: towards high-quality cure of all patients. Haematologica 2007;92(11):1519-32.

4. Zanichelli M.A. Perspectivas para a leucemia mielóide aguda na infância após a observação de um grupo de pacientes tratados convencionalmente. Rev Bras. Hematol. Hemoter 2006;28(4):246-52.

5. Chang M.R., et al., Study of 30 cases of histoplasmosis observed in the Mato Grosso do Sul State, Brazil. Rev Inst Med Trop Sao Paulo 2007;49(1):37-9.

6. Wheat L.J., et al. Clinical practice guidelines for the management of patients with histoplasmosis: 2007 update by the Infectious Diseases Society of America. Clin Infect Dis 2007;45(7):807-25.

7. Fulkerson W.A., B.W. Davis. Opportunistic infections in chronic lymphocytic leukemia. South Med J 1979;72(11):1487-8.

8. Schestatsky P., et al. Isolated central nervous system histoplasmosis in immunocompetent hosts: a series of 11 cases. Scand J Infect Dis 2006;38(1):43-8.

9. Unis G., M. Oliveira F., L.C. Severo. Disseminated histoplasmosis in Rio Grande do Sul. Rev Soc Bras Med Trop 2004;37(6):463-8.

10. Wheat L.J., B.E. Batteiger, B. Sathapatayavongs. Histoplasma capsulatum infections of the central nervous system. A clinical review. Medicine (Baltimore). 1990;69(4):244-60.

11. Odio C.M., et al. Disseminated histoplasmosis in infants. Pediatr Infect Dis J 1999;18(12):1065-8.

12. Wheat L.J., C.E. Musial, E. Jenny-Avital. Diagnosis and management of central nervous system histoplasmosis. Clin Infect Dis 2005;40(6):844-52. 